\title{
IMPROVING NEAREST NEIGHBOUR SEARCH IN 3D SPATIAL ACCESS METHOD
}

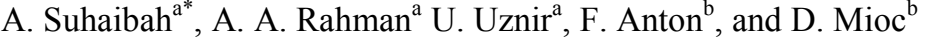 \\ ${ }^{\mathrm{a}}$ Geospatial Information Infrastructure $\left(\mathrm{GeoI}^{2}\right)$ Research Lab., \\ Faculty of Geoinformation and Real Estate, \\ Universiti Teknologi Malaysia, \\ 81310 Skudai, Johor, Malaysia \\ norsuhaibah@gmail.com,mduznir@utm.my, alias@utm.my \\ ${ }^{\mathrm{b}}$ Dept. of Geodesy, National Space Institute, Technical University of Denmark, \\ Elektrovej 328, 2800 Kgs. Lyngby, Denmark \\ fa@space.dtu.dk, mioc@space.dtu.dk
}

Commission II, WG II/2.

KEY WORDS: Nearest Neighbour, 3D Data Clustering, 3D Spatial Access Method, 3D GIS, Data Management, Information Retrieval

\begin{abstract}
:
Nearest Neighbour $(\mathrm{NN})$ is one of the important queries and analyses for spatial application. In normal practice, spatial access method structure is used during the Nearest Neighbour query execution to retrieve information from the database. However, most of the spatial access method structures are still facing with unresolved issues such as overlapping among nodes and repetitive data entry. This situation will perform an excessive Input/Output (IO) operation which is inefficient for data retrieval. The situation will become more crucial while dealing with 3D data. The size of 3D data is usually large due to its detail geometry and other attached information. In this research, a clustered 3D hierarchical structure is introduced as a 3D spatial access method structure. The structure is expected to improve the retrieval of Nearest Neighbour information for 3D objects. Several tests are performed in answering Single Nearest Neighbour search and $k$ Nearest Neighbour $(k N N)$ search. The tests indicate that clustered hierarchical structure is efficient in handling Nearest Neighbour query compared to its competitor. From the results, clustered hierarchical structure reduced the repetitive data entry and the accessed page. The proposed structure also produced minimal Input/Output operation. The query response time is also outperformed compared to the other competitor. For future outlook of this research several possible applications are discussed and summarized.
\end{abstract}

\section{INTRODUCTION}

Nearest neighbour analysis has always been an interesting topic in computer science and geospatial science. The importance of nearest neighbour information could be seen in various applications such as image processing, pattern recognition and Deoxyribonucleic Acid (DNA) sequencing. Numerous models and techniques have been developed due to its popularity and potential. In spatial databases, spatial access methods are used to index and refine the search area to process nearest neighbour queries. Most of the work or research on nearest neighbour search is to focus on query point $q$, and retrieve $k$ nearby objects from a set of points $P$ with respect to the Euclidean distance or a network distance.

In order to efficiently retrieve the nearest neighbour information, we proposed a spatial access method known as clustered hierarchical structure. This structure is constructed based on group clustering and transformed into a hierarchical structure. Based on our previous work (Azri et al., 2015; Azri et al., 2014), the structure produced a minimal coverage and overlap percentage among nodes to avoid repetitive data entry. Thus, in this paper, we proposed the clustered hierarchical structure in order to retrieve nearest neighbour information. The construction of hierarchical structure has been discussed in our previous work. However, our focus in this paper is to test the developed structure in retrieving the nearest neighbour information.

This paper is organized as follows: problems and motivation regarding the nearest neighbour information retrieval in 3D database are discussed in the next section. In Section 3, the concept of the proposed method is explained with its implementation. Section 4 presents the analysis and results of the experiment. Finally, the conclusions are presented in Section 5 .

\section{NEAREST NEIGHBOUR SEARCH USING SPATIAL ACCESS METHOD}

The significance of nearest neighbour information for the marketing and business plan has been mentioned in several researches such as (Akhbari et al., 2014; Kiekintveld et al., 2007; Rodger, 2014). From this information, a lot of analysis can be done such as identifying the best vehicle routing for products delivery, strategize the target group of nearby 
residential and many more. In (Boonsam et al., 2011), nearest neighbour information is used to schedule the tour procedure for transporting the products from one centre to another.

According to the first law of geography, everything is related to everything else, but near things are more related than distant things. Thus, inaccurate results will not optimize the business and marketing strategy. Considering the business of franchising and chain stores in urban areas with high rise multi-level buildings, a $3 \mathrm{D}$ method is prominently required in order to locate and identify the nearest neighbour information accurately. $3 \mathrm{D}$ information is required in the process of identification such as at which level of the franchise unit will be located or is the franchise unit located is at the best level for visibility purposes. However, with the immense number of urban datasets, the analysis and the efficiency of nearest neighbour information retrieval will become more complex and crucial.

In a database management system (DBMS) environment, several tree structures are used to constellate data and information such as R-Tree (Guttman, 1984), Quad Tree, BSPtree and $k \mathrm{~d}$-Tree. However, these structures are still facing the issue of overlap between nodes. For instance, Oracle Database provides a 3D R-Tree structure to deal with 3D data (Murray, 2009; Ravada et al., 2009). However, when the R-Tree is extended into 3D space, the MBV of sibling nodes tends to frequently overlap, and MBVs among nodes can even contain other MBVs. In spatial databases, overlap between nodes is the main reason for the low efficiency of queries due to multi-path queries.

Due to critical overlap of sibling nodes and uneven size of nodes in 3D R-Trees, a research was conducted in (Zhu et al., 2007) to minimize the overlap and optimize the clustering algorithm by introducing $k$-means clustering algorithm to put forward an improved 3D R-Tree. From this experiment, by using an improved algorithm, the overlapping of node is minimized while balancing the volume of parallelepipeds. However, using $k$-means would not drastically minimize the overlap among nodes. This is due to the random selection of the initial seed or cluster centre which will lead to unbalance number of points in each cluster. This condition will increase the risk of having serious overlap in the tree structure. Thus, an improved method of clustering is needed to push the limits of the 3D R-Tree structure in order to access nearest neighbour information.

\section{CLUSTERED HIERARCHICAL TREE STRUCTURE}

\subsection{Clustering Algorithm}

In our previous work (Azri et al., 2015; Azri et al., 2014) clustered hierarchical structure is developed based on partitionbased clustering as a clustering algorithm to group the objects. Partition-based clustering is a method of clustering that requires a pre-set number of clusters from the user. The commonly used algorithm from this type of cluster is $k$-means algorithm. By using this algorithm, data will be partitioned into $k$ clusters $\left(C_{1}\right.$, $C_{2}, \ldots, C_{k}$ ). The means for all instances in each cluster are then calculated as cluster centres.
The linear complexity is one of the reasons of $k$-means popularity. Even if the size of instances is large, this algorithm is computationally attractive. Besides that, the $k$-means algorithm offers simplicity, speed and adaptability to sparse data (Dhillon and Modha, 2001). However, there is a function in kmeans algorithm that is inclined to focus cluster centres at one point or area. Thus, any selection is very sensitive to the group cluster and may make a difference for the end result.

To overcome this issue, clustered hierarchical tree used the $k$ means++ algorithm invented by (Arthur and Vassilvitskii, 2007). The $k$-means++ algorithm is a variant of $k$-means algorithm. It chooses cluster centres by using weighted squared distance from the closest cluster centres. The initial seed of cluster centres are defined and combined with the $k$-means algorithm. The algorithm of $k$-means++ is described as follows.

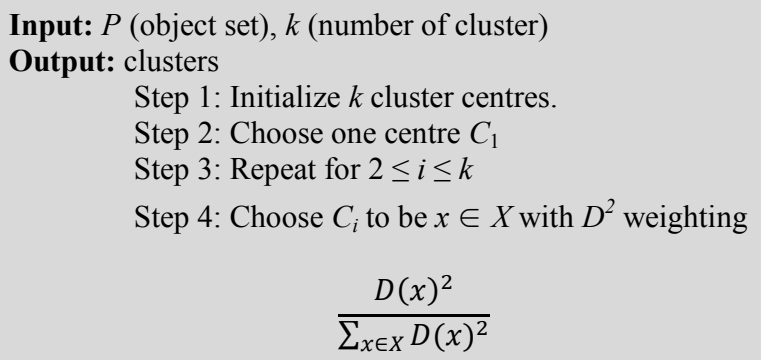

Step 5: while termination condition is not satisfied do Step 6: Assign objects to the nearest cluster centre. Step 7: Update cluster centres. Step 8: end while

From the clusters, hierarchical structure will be constructed. Each parallelepiped is assigned as a child node to the tree. The parent node is the constructed parallelepiped based on minimum value of $x_{\min }, y_{\min }$ and $z_{\min }$ of point location for all clusters and maximum value of $x_{\max }, y_{\max }$ and $z_{\max }$ of point location for all clusters. Parent and child nodes for clustered hierarchical tree structure are described in Figure 1.

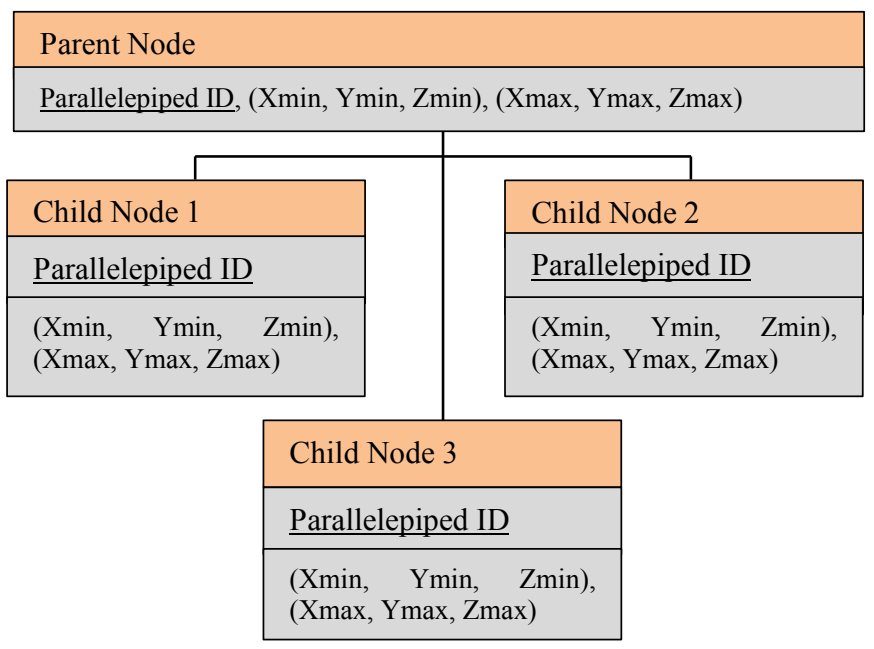

Figure 1. Clustered Hierarchical Tree Structure 
Based on our previous work, minimum coverage and overlap percentage among nodes can be achieved from the clustered hierarchical structure. Table 1 shows the comparison of overlap percentage between partition-based clustering hierarchical structure and 3D R-Tree structure. From the table, proposed clustered hierarchical tree structure produce minimum overlap percentage. Eventhough the percentage is linearly increased each time the number of dataset grew, the structure still keeping it minimum compared to $3 \mathrm{D}$ R-Tree.

Table1. Overlap percentage analysis for varying number of datasets (Azri et al., 2015)

\begin{tabular}{l|c|c}
\hline \multicolumn{3}{|c}{ Percentage of Overlap (\%) } \\
\hline $\begin{array}{l}\text { No. of } \\
\text { Datasets }\end{array}$ & $\begin{array}{c}\text { Clustered Hierarchical } \\
\text { Tree }\end{array}$ & 3D R-Tree \\
\hline $\mathbf{1 0 0 , 0 0 0}$ & $68 \%$ & $92 \%$ \\
$\mathbf{3 0 0 , 0 0 0}$ & $72 \%$ & $95 \%$ \\
$\mathbf{5 0 0 , 0 0 0}$ & $75 \%$ & $97 \%$ \\
\hline
\end{tabular}

\subsection{Nearest Neighbour Query using Clustered Hierarchical}

The nearest neighbour information can be retrieved by traversing the hierarchical structure from its parent node. The traversal started by identifying the location of point query $q$. The location of point query $q$ will serve to identify to which cluster it lies. Other clusters will be pruned to refine the search area. The nearest object with minimum distance $D_{\min }$ will be identified as a nearest object to point query $q$. In this paper, two types of nearest neighbour query are presented. The queries are Single Nearest Neighbour Search and $k$ Nearest Neighbour $(k \mathrm{NN})$.

\subsubsection{Single Nearest Neighbour Search}

Given a set of data points $P$ and a query point $q$, and an integer $k=1$, the nearest neighbour query is to find a single point such that for any $p \in(P-k \mathrm{NN})$ and any $p^{\prime} \in k \mathrm{NN}$, dist $\left(p^{\prime}, q\right) \leq$ $\operatorname{dist}(p, q)$.

Nearest neighbour identification can be performed using clustered hierarchical tree structure. The structure will identify the location of point query $q$ from SQL (Structured Query Language) statement. Then it will identify the group of clustering. After this process the search area is refined. Points in the search area are the candidate point $p$ of nearest neighbour. Since the data has been clustered the candidate point $p$ has been minimized and Input/Output to the page access is decreased. The overall workflow in finding single nearest neighbour is presented in the Figure 2.

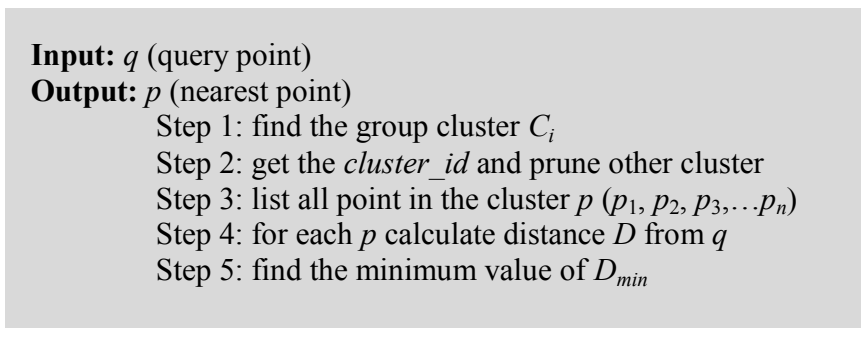

Figure 2. Single Nearest Neighbour workflow

\subsection{2 $k$ Nearest Neighbour $(k N N)$ Search}

$k$ Nearest Neighbour $(k \mathrm{NN})$ query is a type of query that search $k$ number of nearest neighbour from query point $q$. There are a lot of potential applications that can be used together with $k \mathrm{NN}$ query. Consider, for example, a set of building in urban area. $k \mathrm{NN}$ query can be issued to find several the post offices from a building point $P$. The following definition defined the $k \mathrm{NN}$ query of point $q$.

Given a set of data points $P$ and a query point $q$, and an integer $k>0$, the $k$ nearest neighbour $(k \mathrm{NN})$ query is to find a set of $k \mathrm{NN}$ that consist of $k$ data points such that for any $p \in$ $(P-k N N)$ and any $p^{\prime} \in k N N$, dist $\left(p^{\prime}, q\right) \leq \operatorname{dist}(p, q)$.

Clustered hierarchical tree structure used BFS (Best First Search) algorithm to retrieve $k \mathrm{NN}$ of point $q$. BFS will visit the parent node of the structure and identify potential group cluster of $k \mathrm{NN}$. By exploring all the entries in the cluster, each point and minimum distance to point $q$ are listed in minheap $H$ table. Figure 3 describes the overall workflow of $k \mathrm{NN}$ search using clustered hierarchical tree structure.

\section{Input: $q$ (query point)}

Output: $p$ (nearest point)

Step 1: find the group cluster $C_{i}$

Step 2: get the cluster id and prune other cluster

Step 3: list all point in the cluster $p\left(p_{1}, p_{2}, p_{3}, \ldots p_{n}\right)$

Step 4: for each $p$ in the cluster get the minimum distance $\mathrm{D}$ to $q$

Step 5: list in the minheap $H$

Step 6: return the candidate list in ascending order to point $q$

Step 7: find the best distance of $k$ th closest point to $q$

Figure 3. $k$ Nearest Neighbour workflow

\section{EXPERIMENT AND ANALYSIS}

In this section, clustered hierarchical structure is tested with two main tests in retrieving nearest neighbour information. The first test is analysis of page access by clustered hierarchical structure and its competitor. The second test is query response time analysis. All tests are run on a windows operating system with single Intel Xeon running at $2.2 \mathrm{GHz}$ and $4 \mathrm{~GB}$ Random Access Memory (RAM). 


\subsection{Test 1: Accessed Page of Nearest Neighbour Retrieval}

To test the efficiency of clustered hierarchical structure in retrieving 3D nearest neighbour information, a set of 500,000 point locations in $3 \mathrm{D}$ is utilised in this test. To compare the efficiency of proposed structure, the result is then compared with its competitor, 3D R-Tree structure. 3D R-Tree is a hierarchical based structure invented by Guttman in 1984 (Guttman, 1984). Most of commercial software such as Oracle used this structure to constellate data in the database. To compare both approaches, different values of $k$ range from 1 to 250 are used for the nearest neighbour query. The results are plotted in the Figure 4 as $k$ (the number of nearest neighbours) versus the number of pages. From the plotted results, both approaches shows that the number of pages grew linearly each time the number of nearest neighbours increased. However, number of accessed page using clustered hierarchical tree structure is minimal compared to $3 \mathrm{D}$ R-Tree. Clustered hierarchical tree structure used clustering approach to minimize overlap among nodes. This approach reduces repetitive data entry and multipath query. Thus the accessed page is minimal while retrieving the nearest neighbour information.

\subsection{Test 2: Nearest Neighbour Query Response Time Analysis}

The nearest neighbour data retrieval is also tested for the data response time analysis. Since this hierarchical structure improves the data retrieval capabilities, a group of $k$ nearest neighbour search is performed in order to show the improvement. In this test, the search operation is performed based on cluster id and its location. Data retrieval is measured in millisecond (ms). From the result in Figure 5, the proposed clustered hierarchical tree structure offers a lower data retrieval time compared with hierarchical structure of 3D R-Tree, which is $15 \%$ to $20 \%$ faster.

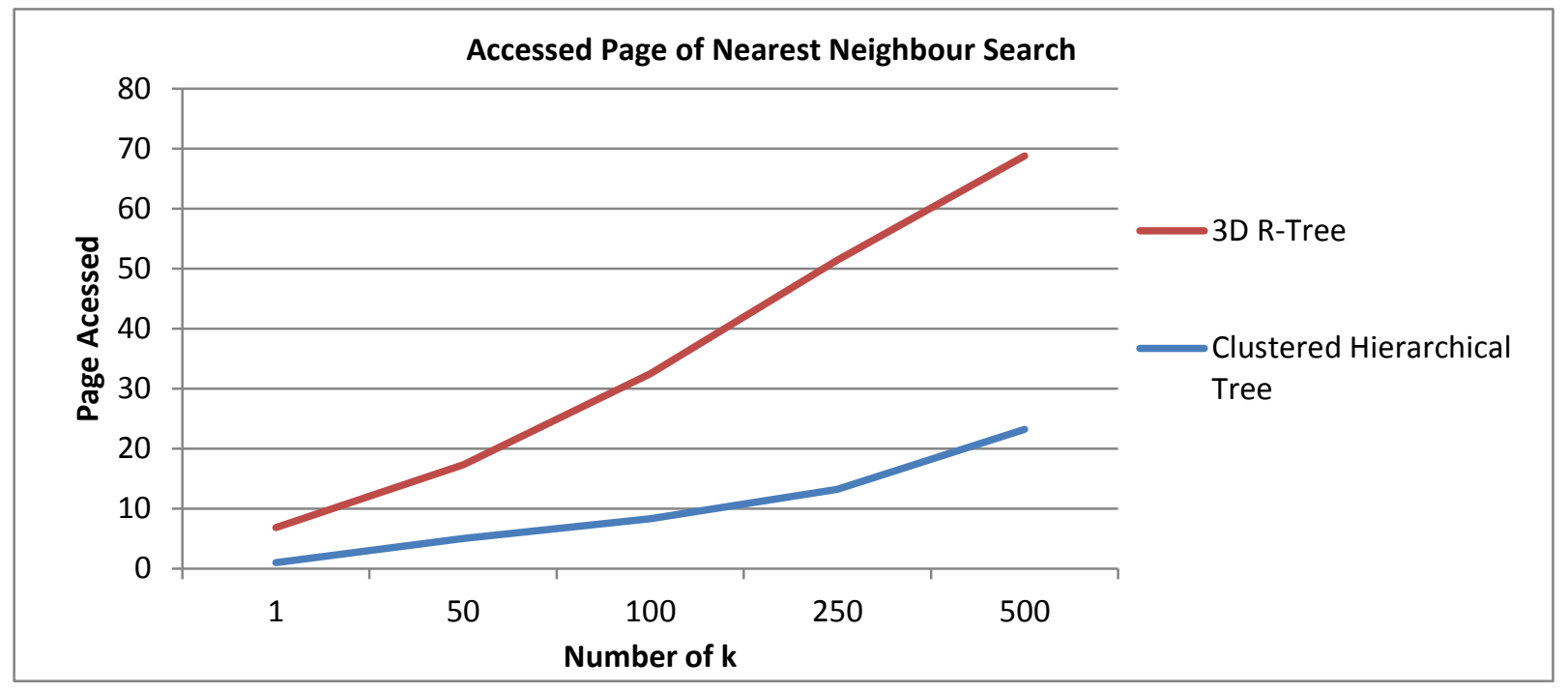

Figure 4. Accessed page of nearest neighbor search

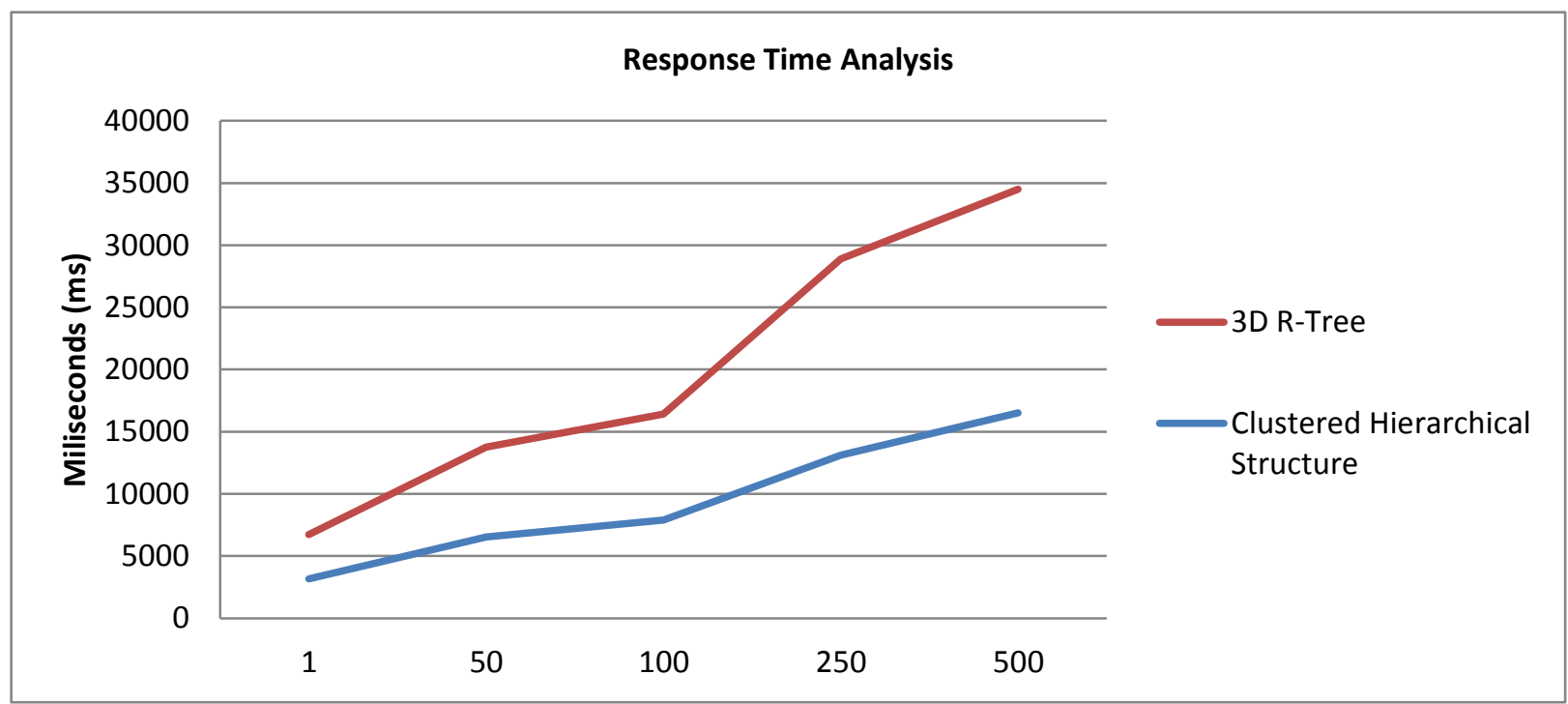

Figure 5. Response time analysis of nearest neighbor search. 


\section{CONCLUSIONS}

This paper proposed a structure of clustered hierarchical tree to constellate 3D data in geospatial databases for efficient nearest neighbour search. The structure is constructed based on clustering algorithm and offers minimal overlap among nodes. Based on our finding, clustered hierarchical tree structure is efficient in handling nearest neighbour search. In the first test, it is proved that the structure has a minimal accessed to the disk page while retrieving the information. Due to this advantage, the structure is expected to reduce the time of data retrieval during the search operation. This hypothesis is proved by performing the second test; Nearest Neighbour Query Response Time where the time is $15 \%$ to $20 \%$ faster than its competitor.

For future outlook, clustered hierarchical tree structure is expected to be applied in several applications such as profilebased marketing where information of surrounding neighbours is important. The neighbouring information could be used in marketing plan or sales analysis. Another possible application is urban planning where most of the analyses require neighbouring information. Other application such as analysis of protein cells in bioinformatics could also be a potential framework for the implementation of clustered hierarchical tree structure.

\section{ACKNOWLEDGEMENT}

This work is supported by the Ministry of Education, Malaysia under the MyPhD, MyBrain15 initiatives and UTM Research University Grant, Vote J.130000.2427.02G77.

\section{REFERENCES}

Akhbari, M., Zare Mehrjerdi, Y., Khademi Zare, H., Makui, A., 2014. A Novel Continuous kNN Prediction Algorithm to Improve Manufacturing Policies in a VMI Supply Chain. International Journal Of Engineering, Transactions B: Applications 27, 1681-1690.

Arthur, D., Vassilvitskii, S., 2007. K-Means++: The Advantages Of Careful Seeding, Proceedings Of The Eighteenth Annual ACM-SIAM Symposium on Discrete Algorithms. Society for Industrial and Applied Mathematics, Pp. 1027-1035.

Azri, S., Anton, F., Ujang, U., Mioc, D., Rahman, A., 2015. Crisp Clustering Algorithm for 3D Geospatial Vector Data Quantization, In: Breunig, M., Al-Doori, M., Butwilowski, E., Kuper, P.V., Benner, J., Haefele, K.H. (Eds.), 3D Geoinformation Science. Springer International Publishing, Pp. 71-85.

Azri, S., Ujang, U., Rahman, A.A., Anton, F., Mioc, D., 2014. Spatial Access Method for Urban Geospatial Database Management: An Efficient Approach of 3D Vector Data Clustering Technique, 2014 9th International Conference on Digital Information Management, ICDIM 2014, Pp. 92-97.

Boonsam, P., Suthikarnnarunai, N., Chitphaiboon, W., 2011. Assignment Problem and Vehicle Routing Problem for an Improvement of Cash Distribution, Proceedings of The World Congress on Engineering and Computer Science, Pp. 11601164.
Dhillon, I.S., Modha, D.S., 2001. Concept Decompositions for Large Sparse Text Data Using Clustering. Mach. Learn. 42, 143-175.

Guttman, A., 1984. R-Trees: A Dynamic Index Structure for Spatial Searching. Sigmod Rec. 14, 47-57.

Kiekintveld, C., Miller, J., Jordan, P.R., Wellman, M.P., 2007. Forecasting Market Prices in A Supply Chain Game, Proceedings of The International Conference on Autonomous Agents, Pp. 1323-1330.

Murray, C., 2009. Oracle Spatial Developer's Guide, 11g Release 1 (11.1), Oracle Spatial User's Guide And Reference, Pp. 1 - 870.

Ravada, S., Kazar, B., Kothuri, R., 2009. Query Processing In 3D Spatial Databases: Experiences With Oracle Spatial 11g, In: Lee, J., Zlatanova, S. (Eds.), 3D Geo-Information Sciences. Springer Berlin Heidelberg, Pp. 153-173.

Rodger, J.A., 2014. A Fuzzy Nearest Neighbor Neural Network Statistical Model for Predicting Demand for Natural Gas and Energy Cost Savings in Public Buildings. Expert Syst. Appl. 41, 1813-1829.

Zhu, Q., Gong, J., Zhang, Y., 2007. An Efficient 3D R-Tree Spatial Index Method for Virtual Geographic Environments. ISPRS Journal Of Photogrammetry And Remote Sensing 62, 217-224. 